A Mode of producing Arago's Rotation

This article has been downloaded from IOPscience. Please scroll down to see the full text article.

1879 Proc. Phys. Soc. London 3115

(http://iopscience.iop.org/1478-7814/3/1/318)

View the table of contents for this issue, or go to the journal homepage for more

Download details:

IP Address: 128.226.37.5

The article was downloaded on 06/05/2013 at 14:07

Please note that terms and conditions apply. 
IV. By turning the graduated circle with the arc $\mathrm{T}$ and the arm I attached to it on the face of the fixed vertical circle (i.e. round a horizontal axis perpendicular to the plane of the optic axes), and reading the verniers on both sides of the circle, the angles between the directions of the optic axes may be accurately measured.

\section{A Mode of producing Arago's Rotation.}

\section{By Walter Baily, M.A.*}

[Plates XV. \& XVI.]

ARAGo's method of producing rotation in a copper disk consists of suspending it by its centre so as to make it lie horizontally above the poles of a horseshoe magnet, and then rotating the magnet about a vertical axis. The rotation of the disk is due to that of the magnetic field in which it is suspended; and we should expect that if a similar motion of the field could be produced by any other means, the result would be a similar motion of the disk.

Possibly the rotation of the magnet may be the only practicable way of producing a uniform rotation of the field; but it will be shown in this paper that the disk can be made to rotate by an intermittent rotation of the field effected by means of electromagnets.

Suppose two magnetic poles to be below a plane sheet of a conducting substance capable of moving in its own plane. Each pole may be regarded as a small circular current parallel to the disk. The currents will be in the same or different directions according as the poles are of the same or different names. We will examine the effect of a change in the strength of either of the poles, in giving the sheet a tendency to move.

There are four cases ; viz.-

1. Poles alike. One increasing.

2. " " One diminishing.

3. Poles unlike. One increasing.

4. ", " One diminishing.

In case (1) the increasing pole induces in the portion of the sheet opposite to itself a circular current opposite in direction 
Proc Phys. Soc Vol. 3 . Pl.XV

Fig. 5.

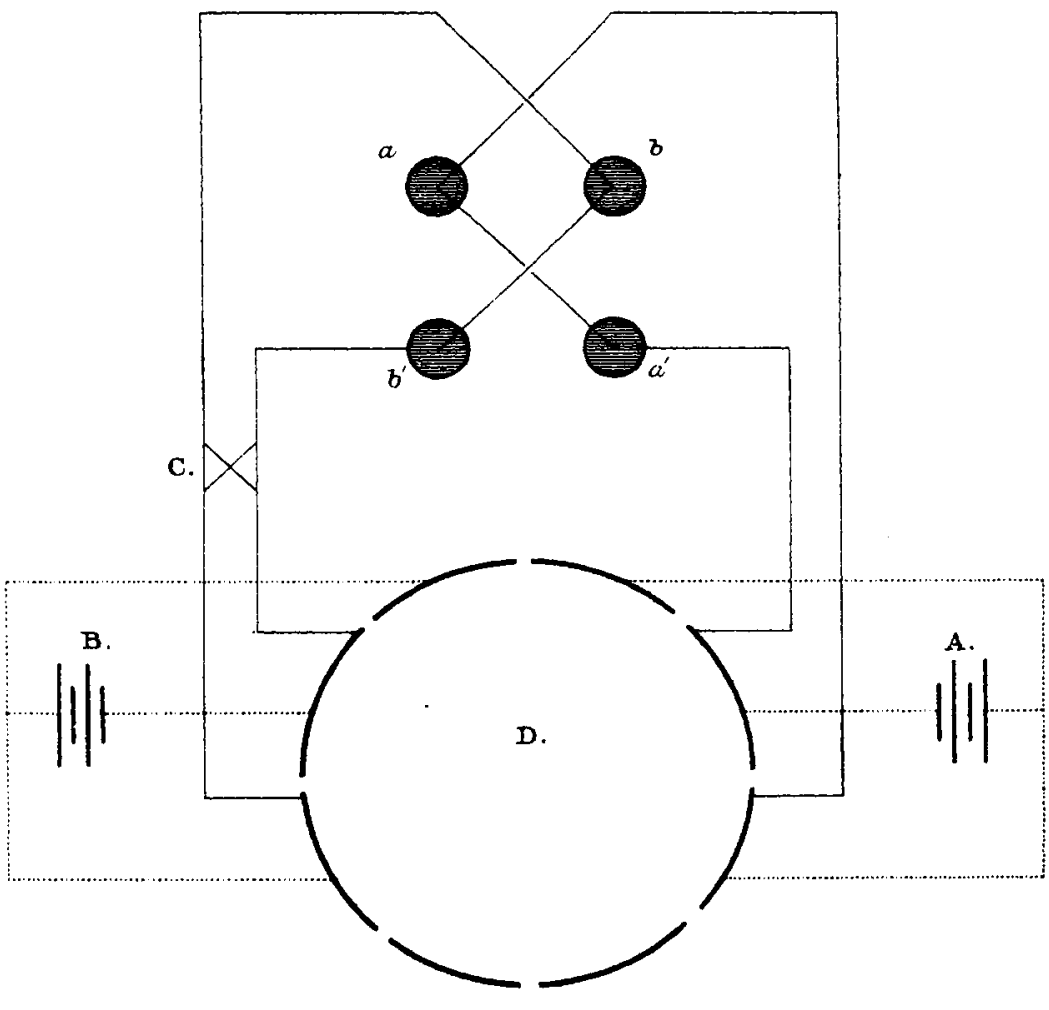

Fig. 6.

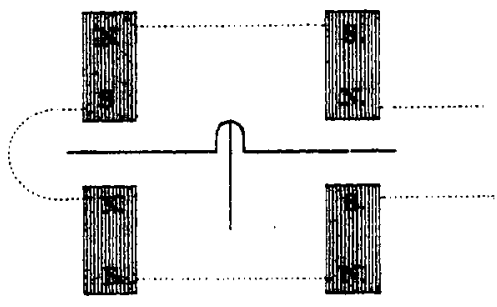



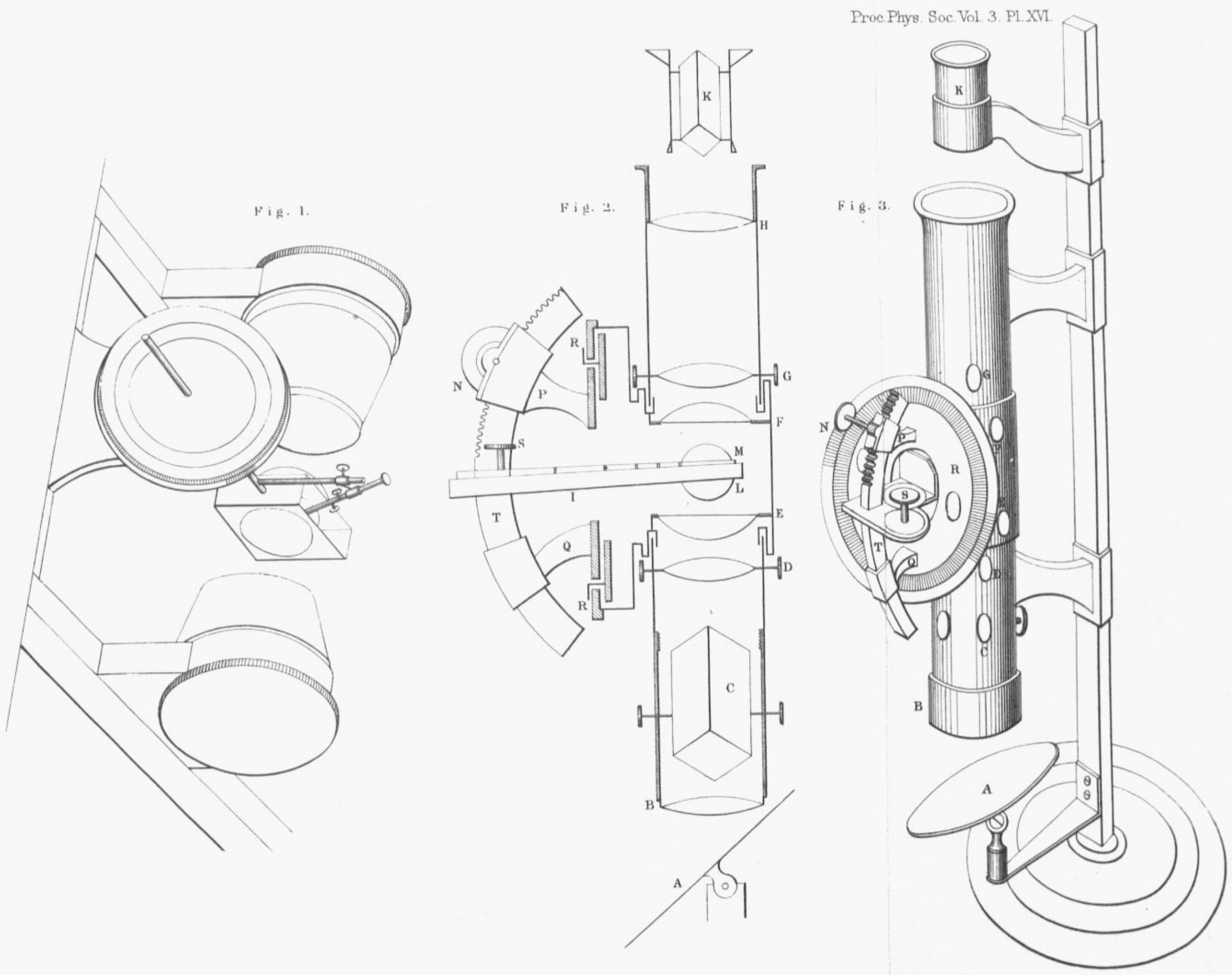
to the currents representing the poles. Hence this portion of the sheet is repelled by both poles. The repulsion from the increasing pole is perpendicular to the sheet, and gives it no tendency to move; but the repulsion from the other pole tends to move the sheet from the constant pole toward the one which is increasing.

In case (2) the diminishing pole induces in the portion of the sheet opposite to it a circular current in the same direction as those representing the poles; so that this portion of the disk is attracted by both poles, and the disk tends to move from the diminishing pole toward the one which is constant.

In cases (3) and (4) it can easily be shown that the results are the opposite to those obtained in cases (1) and (2) respectively.

If one pole increases while the other diminishes, both tendencies to move are in the same direction, and the resulting tendency is the sum of the two.

The pole of an electromagnet made or broken is the extreme case of a pole increasing or diminishing.

Now conceive an even number of vertical bar electromagnets arranged in a circle with their upper poles in one horizontal plane, and a copper disk to be suspended above them; and the two magnets at the extremities of each diameter to be coupled together, and with a battery, so that each such pair of magnets forms a horseshoe electromagnet independent of all the others. Let $P, Q, R, S$ be pairs of magnets at the ends of successive diameters. Make $P$, and then make $Q$ so that the north pole of $Q$ is adjacent to the north pole of $P$, and therefore the south pole of $Q$ adjacent to the south pole of $P$. Then by case (1) the portion of the disk opposite the north pole of $P$ is driven towards the north pole of $Q$; and a similar action takes place at the south poles. Now break $P$. By case (2) the portion opposite the north pole of $P$ is again driven towards the north pole of $Q$, and so with the south poles. Continuing the action by making $R$ and then breaking $Q$, making $S$ and then breaking $R$, and so on, in each case making the adjacent poles similar, we get a series of impulses on the disk all tending to make it move in one direction round the axis of suspension. Hence the disk will rotate as in Arago's experiment. 
In one extreme case, viz. when the number of electromagnets is infinite, we have the case of a uniform rotation of the magnetic feld, such as we obtain by rotating permanent magnets.

In the other extreme case the number of pairs of electromagnets is reduced to two, and the number of batteries is also reduced to two. Let the poles of one pair of magnets be called $a, a^{\prime}$, and those of the other $b, b^{\prime}$. Then the arrangement of the pole as seen from above is $\frac{a b}{b^{\prime}} a^{\prime}$; and the successive states of these poles will be

$\begin{array}{ccccccccc}1 . & 2 . & 3 . & 4 . & 5 . & 6 . & 7 . & 8 . & 1 . \\ \text { NN } & \text { ON } & \text { SN } & \text { SO } & \text { SS } & \text { OS } & \text { NS } & \text { NO } & \text { NN } \\ \text { SS } & \text { SO } & \text { SN } & \text { ON } & \text { NN } & \text { NO } & \text { NS } & \text { OS } & \text { SS }\end{array}$

$\mathrm{N}, \mathrm{S}$, representing north and south poles respectively, and $\mathrm{O}$. representing that the pole is not magnetic. It appears that the change from 1 to 3 through 2 is nothing more than reversing $a a^{\prime}$; and the change from 3 to 5 consists in reversing $b b^{\prime}$. Similarly we pass from 5 to 7 by reversing $a a^{\prime}$, and again from 7 to 1 by reversing $b b^{\prime}$. The whole process is thus shown to consist in reversing $a a^{\prime}$ and $l b^{\prime}$ alternately.

In passing from 8 to 1 we see by case (1) that the parts of the disk over $b, b^{\prime}$ are respectively repelled from $a, a^{\prime} ;$ and by case (3) we see that the same parts are respectively attracted to $a^{\prime}, a$. Again in passing from 1 to 2 we see by case (2) that the parts above $a, a^{\prime}$ are respectively attracted by $b, b^{\prime}$; and we also see by case (4) that the same parts are respectively repelled by $b^{\prime} b$.

The effect of ench of these two changes is to make the disk tend to rotate in the direction $a b a^{\prime} b^{\prime}$. All the other changes may be shown to have the same effect; 'so that the disk will rotate in the direction $a b a^{\prime} b^{\prime}$.

If starting with the state (1) we roverse $b b^{\prime}$ first, we should have the series of states as follows:-
1.
2.
3.
4.
5.
6. 7.
8. 1.
NN NO NS OS SS SO SN ON NN
SS OS NS NO NN ON SN SO SS

from which it maly be easily shown that the disk would rotate in the direction $b a b^{\prime} a^{\prime}$. 
The experiment with the four electromagnets may be readily performed by means of a commutator which will reverse the currents several times in a second; and a considerable rotation can be given to the disk.

The commutator which I constructed for the experiment consisted of a wheel of wood with a brass rim. This rim was completely cut through in places equidistant from each other; and ten tongues of thin copper pressed against the rim*. These tongues were in two groups of five; and in each group the distances between the contacts of the tongues with the rim were half the distances between the cuts in the rim. The distance between the two groups must be greater than the distance between the contacts.

Let $A$ be $a$ wire from the positive pole of one battery, and $A^{\prime} A^{\prime \prime}$ be wires from the negative pole of the same battery; $B$ a wire from the positive pole of the other battery, and $B^{\prime} B^{\prime \prime}$ wires from the negative pole; and let $a a^{\prime}$ be the ends of the coil round one pair of electromagnets, and $b b^{\prime}$ the ends of the coil round the other pair. In the figures 1 to 4 the wheel is seen in four consecutive positions, with the tongues in contact with it; and the letters show with what wire each tongue is connected.

The contact of two tongues with the same section of the ring puts the wires to them into electric connexion.

The commexions are, in

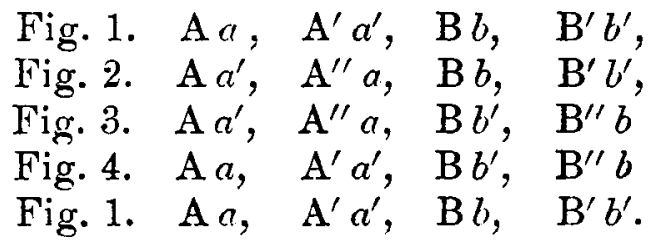

Hence in passing from fig. 1 to fig. 2 the current through $a a^{\prime}$ is reversed; in passing to fig. 3 that through $b b^{\prime}$ is reversed. The current through $a a^{\prime}$ is reversed again in passing to fig. 4, and that through $b b^{\prime}$ is reversed again in passing to fig. 1. The commutator is thus seen to reverse each pair of magnets twice while rotating through the angle subtended by one division of the rim; so that with eight divisions one turn of the wheel reverses each pair of magnets sixteen times.

* The number of cuts must not be less than seven. I nsed eight eits. 
If the wheel is rotated in the opposite direction, the series of magnetic states is obtained in the reverse order, and the disk rotates in the opposite direction. But there is a better method of reversing the motion of the disk-which is, to introduce an ordinary commutator into one of the circuits, either between the battery and the wheel, or between the wheel and the magnets. The reversal of this commutator reverses the motion, while the wheel is rotated continuously in one direction. Fig. 5 gives the arrangement of the connexions. A, B are the batteries; $a, a^{\prime}, b, b^{\prime}$ the electromagnets; $\mathrm{C}$ the ordinary commutator; and $\mathrm{D}$ the wheel.

It should be noted that the rotation of the disk is accompanied by the formation of induced currents whose intensity depends on the velocity of rotation, and whose effect is to diminish the rotation. These opposing currents are got rid of when the disk is suspended as a torsion-balance, and its deflection observed, as the currents will not be formed except when the disk is moving.

The effect on the disk might be much increased by placing four other electromagnets above the disk, each opposite one of the lower magnets, as connected with it, so that the lower pole of the upper magnet should be of the opposite name to the upper pole of the lower magnet. In fig. 6 one pair of magnets is shown with the opposite pair, and the wires connecting them. The disk is seen in section, balanced on a needle-point, between the two pairs of magnets. The other four magnets are not shown in the figure. 